\title{
Knowledge and $(\mathrm{Ab})$ Use in Connection with Novel Psychoactive Substances: A Cross-Sectional Analysis of Psychedelic Users Existing on Online Platforms
}

\author{
Mohammed-Lutfi Al-Imam ${ }^{1} \&$ Ahmed Al-Imam ${ }^{2,3}$ \\ ${ }^{1}$ College of Mechanical Engineering, University of Baghdad, Iraq \\ ${ }^{2}$ Department of Postgraduate Medicine, School of Life and Medical Sciences, University of Hertfordshire, United \\ Kingdom \\ ${ }^{3}$ Department of Anatomy and Cellular Biology, College of Medicine, University of Baghdad, Iraq \\ Correspondence: Dr Ahmed Al-Imam, House 18/5, Al-Akhtal Street, District 318, Al-Adhamyia, 10053, Baghdad, \\ Iraq. E-mail: tesla1452@gmail.com; a.m.al-imam@herts.ac.uk
}

Received: July 25, 2017 Accepted: August 17, 2017 Online Published: September 18, 2017

doi:10.5539/gjhs.v9n11p51 URL: https://doi.org/10.5539/gjhs.v9n11p51

\begin{abstract}
Background: The prevalence of use and misuse of novel psychoactive substances (NPS) has been escalating at an exponential rate in the past two decades. Studies on NPS are highly concentrated in relevance to the developed world, while few have been undertaken in the developing countries.

Materials and Methods: This study is observational and cross-sectional in nature; it relies on a web-based survey distributed to a population of (ab)users of psychedelics and NPS; this community was accessible via dedicated online platforms psychedelics users located on the Facebook social communication medium. The aim of the study is to estimate the level of knowledge and the extent of (mis)use in connection with psychoactive substances, including the novel ones (NPS).

Results: The number of participants who responded to the survey reached 458 responders. The level of knowledge and (ab)use was relatively high. The majority of participating individuals were Caucasians males from the developed world including the US, the UK, India, and western European countries. The primary religious affiliation was either agnosticism or atheism. Approximately, 26\% were diagnosed with the neuropsychiatric condition.

Conclusion: The NPS phenomenon is still a major concern primarily in the developed world. Additional studies are required in virtual and non-virtual populations. The targeted populations should include; students, medical professionals, academics, patients with psychiatric and neurological abnormalities, convicts and criminals, and even terrorists.
\end{abstract}

Keywords: Novel Psychoactive Substances, NPS, cross-sectional studies, surveys and questionnaires, hallucinogens, prevalence, epidemiology, public health, community medicine, students

\section{Background}

Novel psychoactive substances (NPS), also known as research chemicals and designer drugs, poses a major health problem and an economic concern in the developed world (Brew, 2016; Dargan and Wood, 2013). The burden of the prevalence of substance (mis)use and the extent of electronic commerce (e-commerce) phenomenon in the developing world, including the Arab world and the Middle East, has been marginally studied (Al-Imam et al., 2016; Al-Imam et al., 2017; Dargan and Wood, 2012; Wood et al., 2014). A critical question remains unanswered; how to estimate the prevalence of the (ab)use of NPS? Can that be estimated via modalities other than observational studies of the web? The answer is yes; it can be inferred by modes other than web analytics, for example; cross-sectional studies and surveys in a real (non-virtual) population of users, and retrospectively from; seized batches of illicit substances, databases from criminal records, hospital emergency units, border patrol agencies, police departments, and counter-terrorism units. Those modalities of research have been extensively implemented by regulating bodies including; the World Anti-Doping Agency (WADA), the United Nation Office on Drugs and Crime (UNODC), the International Police Organization (INTERPOL), and the European Monitoring 
Centre for Drugs and Drug Addiction (EMCDDA) (Burns, 2014; Ferri et al., 2015; Mackey \& Liang, 2013).

Most of the previous studies used to assess the extent of substance (ab)use were carried out in the western societies and the developed countries, while very few attempts were prosecuted in the developing world, and even fewer attempts were committed for the region of the Middle East and Arabic countries. For instance, the work accomplished by Al-Hemiary and colleagues (Al-Diwan et al., 2015; Al-Hemiary et al., 2014; Al-Hemiary et al., 2015; Al-Hemiary et al., 2016; Al-Imam et al., 2016; Al-Imam, 2017a; Al-Imam, 2017b; Al-Imam et al., 2017; Bigdeli et al., 2013). Nevertheless, the advent of intranet networks and virtual social communication enabled experts to conduct surveys and cross-sectional analyses promptly and efficiently via the surface web (Best et al., 2014; Hirst et al., 2014; Jensen, 2013; Joh et al., 2017; Lin \& Atkin, 2014; Moorhead et al., 2013).

In this study, a cross-sectional analysis will be deployed; it will rely on a web-based survey targeting a population of NPS and psychedelics users on the surface web. The studied population exist virtually on the Facebook social communication medium; The aim is to assess the level of knowledge and the extent of (ab)use in connection with psychoactive substances including novel ones (NPS). Several private groups on Facebook were approached for this purpose

\section{Materials and Methods}

This study has been ethically approved by the institutional review board of the College of Medicine at the University of Baghdad (IRB meeting number 8, on the $20^{\text {th }}$ of December 2016); the study is observational and cross-sectional; it is based on a survey targeting a virtually-placed population of psychedelics and NPS users. Those groups were accessible via an online platform, the Facebook social communication medium. The aim of the study is to conclude an inference in relation to; the level of knowledge and the extent of (ab)use in connection with psychoactive substances; several demographic parameters were consulted.

The questionnaires of the survey were pilot-tested, and it was later transferred into an electronic form (e-form) which was composed of three main sections; demographic parameters of participants (1), knowledge and (ab)use of substances (2), and the smoking, alcohol drinking habits, and other social habits (3). The survey questionnaires were addressing several parameters including; age, gender, handedness, ethnicity, religious affiliation, number of siblings, place of residence, marital and social status, past medical and psychiatric history, the knowledge on the topic of NPS, the knowledge of specific psychoactive substances, the frequency and the chronicity of use of substances, the experienced adverse effects, drinking and smoking habits, and other social practices. The screened parameters may have a differential effect either on the level of knowledge or the extent of (ab)use of traditional as well as novel psychoactive substances.

The e-form was pilot-tested to ensure the absence of any errors and to estimate the average duration of time required to complete the survey. The time necessary to fill the e-survey was estimated to be around six minutes. The survey itself (e-survey) was created with Google Forms; the survey was then distributed via specific groups on Facebook; these groups were private and dedicated for NPS and psychedelics users. Further, the e-survey has been circulated after acquiring an official permission from the administrators of these groups (Facebook, 2017). The members of those groups have been prompted about the nature and the topic of the survey prior to its distribution. Once the survey was distributed, each participant had only one attempt to fill the questionnaire and submit it. All responses were anonymous; these were stored on a 256 bit-encrypted computer implementing firewall technologies. The responses were later downloaded as comma-separated values (.csv) file format, which was converted into a database on Microsoft Excel 2016, upon which analyses were carried out. Alpha $(\alpha)$ value of 0.05 was set as the cutoff margin for statistical significance. The overall level-of-evidence is estimated to be of level-3b in accordance with the system imposed by the Oxford Center for Evidence-Based Medicine (Howick et al., 2011).

\section{Results}

The total number of individuals who responded to the survey was 458; they were a proper representative of the studied population as they accounted for almost a quarter $(26.3 \%)$ of the studied population. The age of the participants was ranging from 14 to 73 years with an average of $28.92(+/-11.83)$ years (Figure 1); the vast majority of participants were aged 18 years $(7 \%)$ and $21(7 \%)$, while those aged 17, 19, and 20 represented $6 \%$ for each. Hence the majority of the population are aged $17-21$ years which constitute $32 \%$ of the total number of responders. Responders aged of 30 and 32 years has $4 \%$ representation for each. It can be assumed that a bimodal peak of age for responders exist (17-21 years and 30-32 years) which sum up to $40 \%$ of the total number of responders. On the other hand. Statistical outliers include responders who are aged 57 years and older. Males were predominant (77.3\%), while females and transgender individuals accounted for $21 \%$ and $1.7 \%$ respectively. The majority were right-handed (88\%), and no ambidextrous individuals were detected. Regarding the sexual 
orientation, most were heterosexual (79.4\%), while homosexual (gay and lesbians) individuals accounted for $2.6 \%$. Bisexual people represented a considerable percentage $(16.9 \%)$, while asexual participants and those with zoophilia represented a minute fraction of $0.9 \%$ and $0.2 \%$ respectively. Caucasians were the predominant ethnic group 82\% (Figure 2), while Hispanic and Latin individuals represented 5.8\% combined. Other ethnicities were minorities including; Asians and Pacific Islanders (5.1\%), Native Americans and American Indians (2.6\%), Arabs (1.9\%), and Blacks and Africans (1.7\%); other ethnicities included Kurdish people although their contribution was negligible. Blacks and Africans also represented a minority although it is well known that the social networking sites are being utilised by African Americans at a higher rate than by the non-Hispanic whites (Chou et al., 2009; Moorhead et al., 2013).

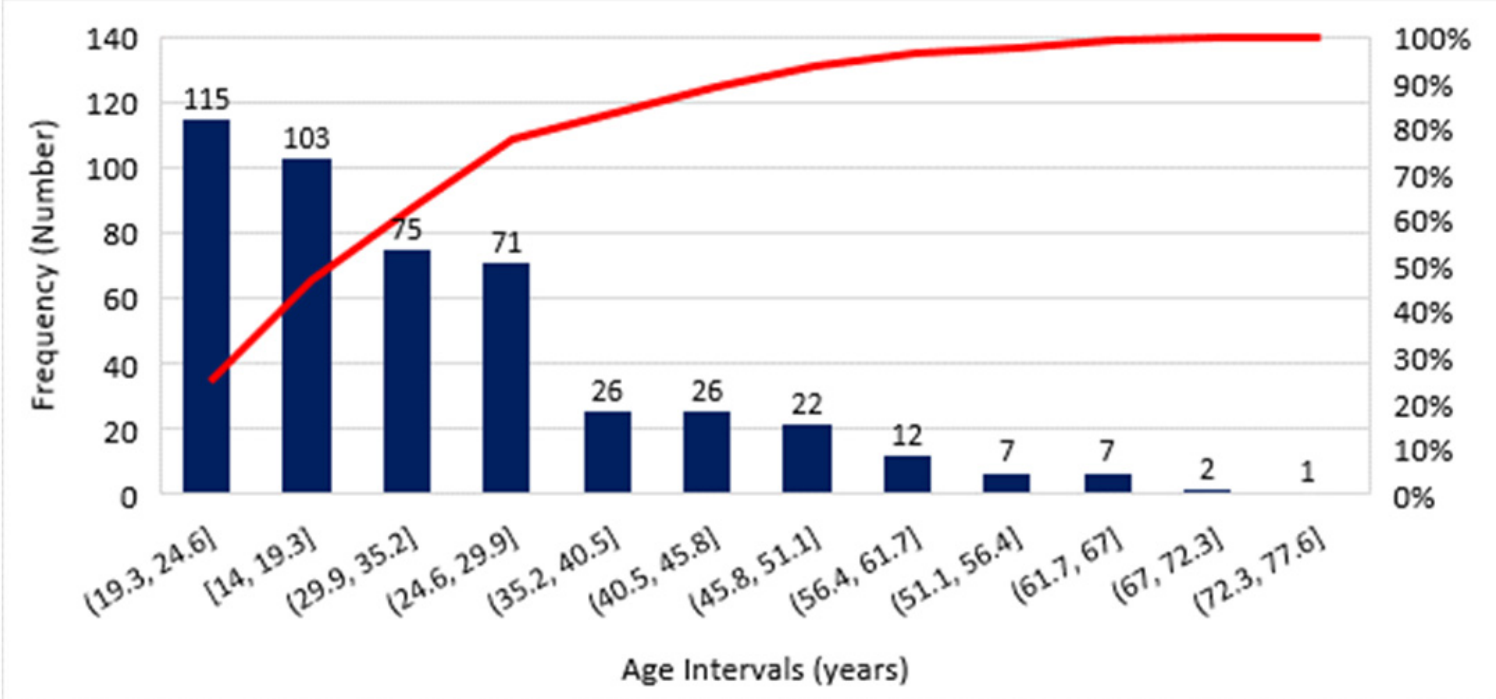

Figure 1. Age of the Responders: Pareto Chart

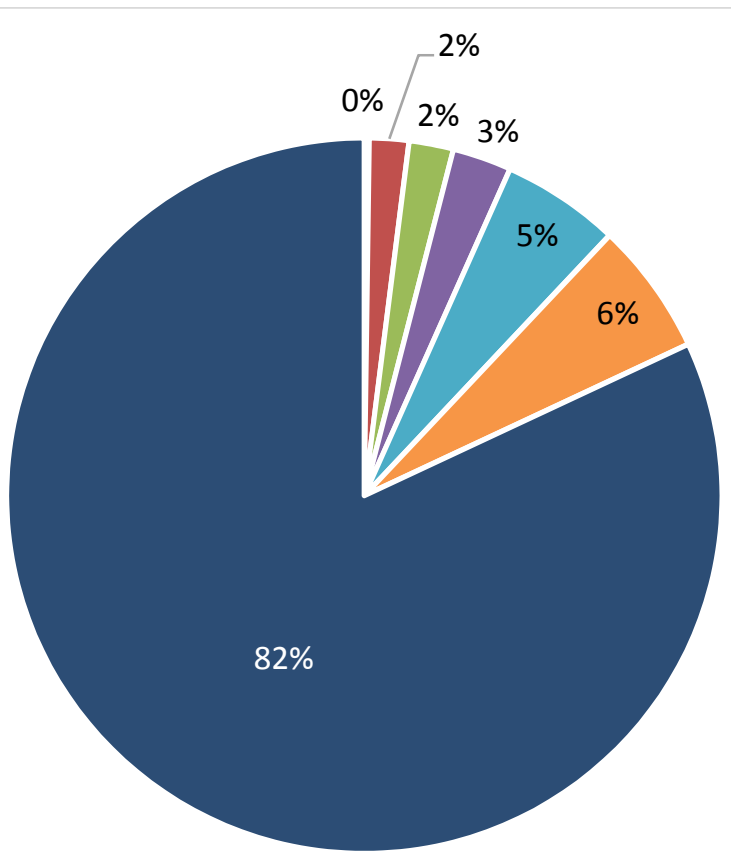

- Kurdish

- Black / African

Arab

- Native American / American Indian

- Asian / Pacific Islander

- Hispanic / Latin

- Caucasian

Figure 2. Ethnic Contribution of the Responders: Pie Chart 
Geographic mapping (geo-mapping) of the participants (Figure 3) shows that the main contributing countries were located in North America, India, and western Europe. In fact, the top ten contributing countries (out of 57 countries) accounted for approximately $80 \%$ of the total. These countries (in descending order of frequency); US, UK, Canada, Australia, India, New Zealand, Sweden, Denmark, Belgium, and France. The Middle East contribution to the geo-map was minimal at $2.36 \%$. Middle Eastern and Arabic countries included (in ascending order of frequency); Iraq, Israel, Morocco, Bahrain, Tunisia, and Turkey. Concerning the religious affiliations (Figure 4), the majority adopted either atheism (29\%) or agnosticism (24\%). Other affiliations included; spiritualism (14\%), pantheism (8\%), and Christianity (8\%). Buddhists and Muslims contributed to $7 \%$ and $3 \%$ respectively, while Pagans, Hindu, and Taoists accounted for $2 \%, 1 \%$, and $1 \%$ respectively. The rest of the religious affiliations lumped for a minority of 3\%. Those responders could be Baha'is, Deist, Jewish, Rastafari, Santo Diame, Shamanist, Yogi, and Theosophy. It is possible that a particular religious affiliation may have a differential effect, in addition to the ethnic and sociocultural norms, on the individual's level of knowledge or the extent of (ab)use of the psychoactive substance, both traditional and novel.

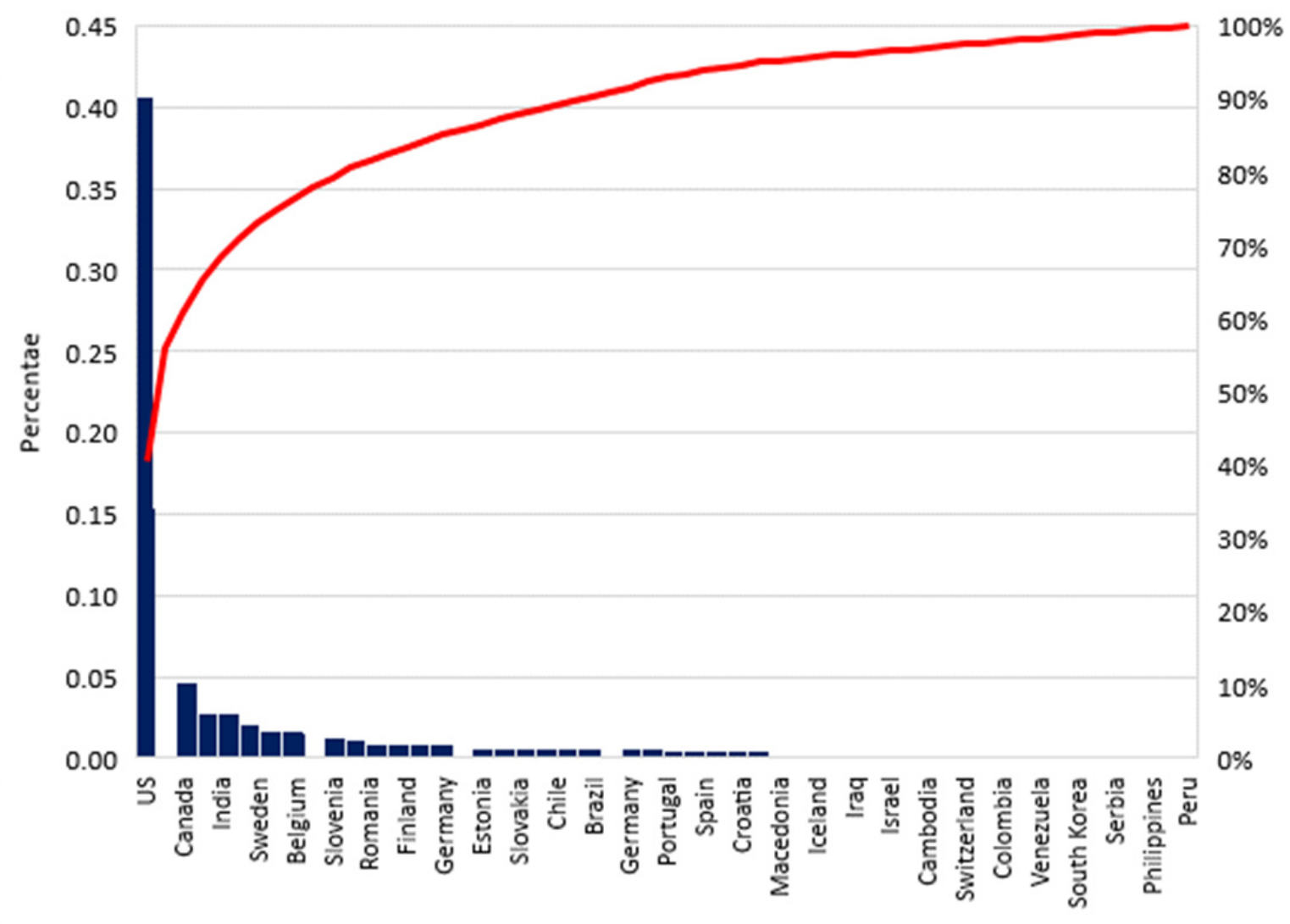

Figure 3. Geographic Mapping (Geo-mapping) of the Responders 


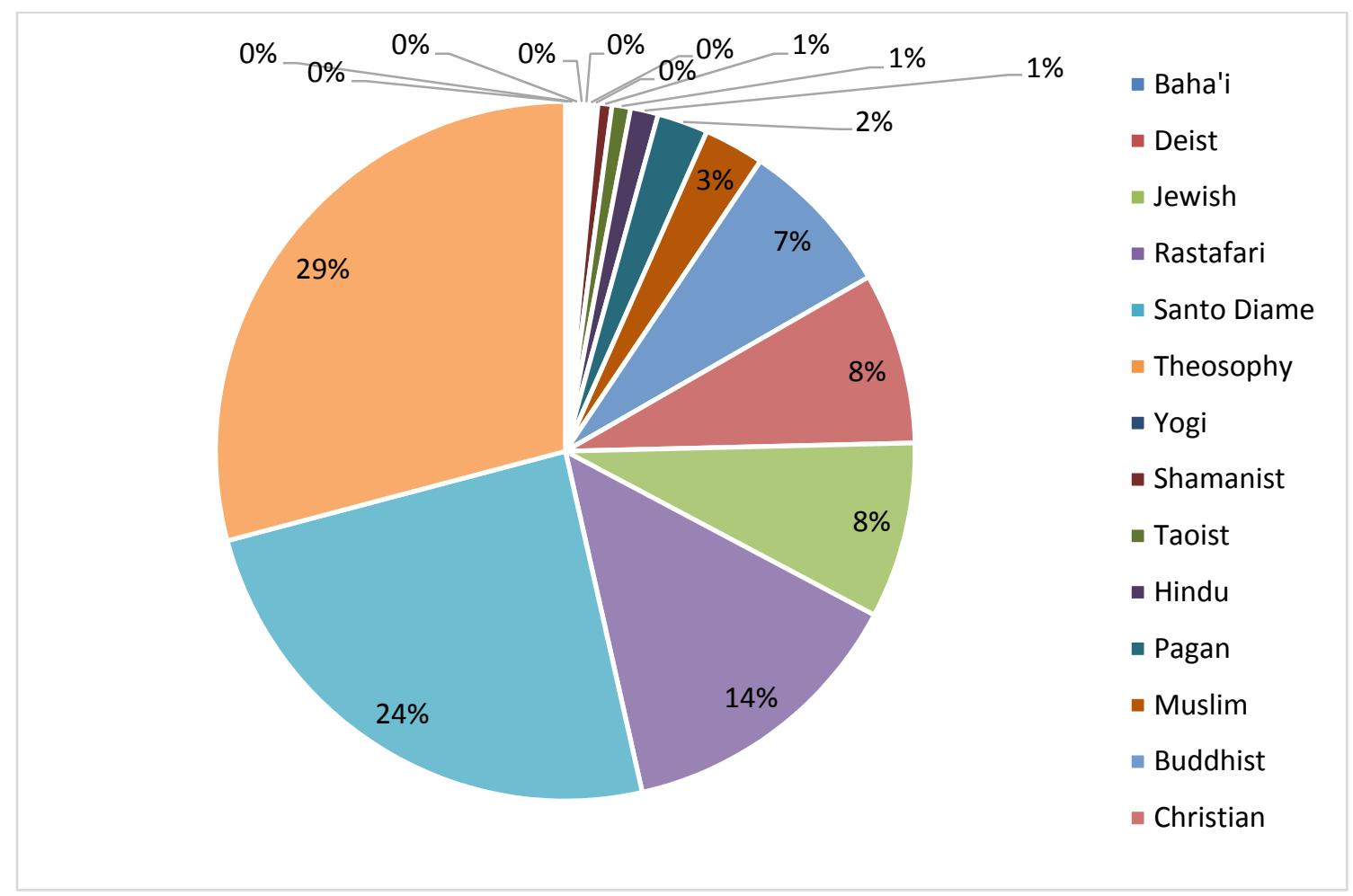

Figure 4. Religious Affiliations of the Responders

Most of the responders were residents of urban areas while rural area residents accounted for $29.1 \%$; the majority of those either lived with their parents $(32.5 \%)$ or with a male/female partner $(30.6 \%)$, while those who lived alone or with friends accounted for 19.3 and $15.8 \%$ respectively, and a minority of participants lived on-campus (1.7\%). A good percentage were unemployed (40.5\%). With regard to their social and marital status, $64.5 \%$ were single, while individuals who were either married or in a de facto relationship accounted for $14.6 \%$ for each. Participants who were either divorced $(5.6 \%)$ or widowed $(0.9 \%)$ made a combined percentage of $6.5 \%$. Only $26.3 \%$ were diagnosed with either neurological or psychiatric condition; most were related to the neurotic spectrum rather than psychotic conditions. Neurotic conditions include; bipolar disorder, personality disorders, anxiety and generalized anxiety disorder (GAD), depression, dysthymia, insomnia, mania, panic disorder, post-traumatic stress disorder (PTSD), attention deficit disorder (ADD) and attention deficit hyperactivity disorder (ADHD), body integrity identity disorder, body dysmorphic disorder, phobias including social phobia and agoraphobia, obsessive-compulsive disorder, dermatillomania, autism, Asperger's syndrome, depersonalization-derealization disorder, obstructive sleep apnea syndrome, dyslexia, dyspraxia, and multiple sclerosis.

Psychotic conditions were less diverse; they included; schizoaffective disorder, schizophrenia, and Dissociative identity disorder. However, those psychotic conditions did not exceed $4 \%$ of the entire spectrum of neuropsychiatric conditions. On the other hand, $32.5 \%$ of the participants disclosed their need to be evaluated by a psychiatrist either for social support or to treat their existing psychiatric condition, while $22.9 \%$ of the participants stated that they have attempted to commit suicide. Approximately $69.4 \%$ were habituated to drink caffeinated beverages (Pepsi, Coca Cola, etc.), and 37\% were habituated to energy drinks, while Smokers accounted for 56.7\%. Participants who drank alcohol accounted for $63.6 \%$, while $62.3 \%$ of the participants confessed that they have suffered from alcoholism and alcohol dependence syndrome (ADS).

In relation to the level of knowledge among (ab)users, $90.6 \%$ of the participants declared their awareness of the terms; novel psychoactive substances, NPS, research chemicals, legal highs, designer drugs, and street drugs. Of those who are knowledgeable of psychoactive substances and NPS, the level-of-knowledge was either negligible $(7.7 \%)$, basic $(31.9 \%)$, intermediate $(39.4 \%)$, or advanced $(21 \%)$. Participants have also identified their source of knowledge to be from the Internet $(81.2 \%)$, friends and family $(52.9 \%)$, drug fora $(33.8 \%)$, textbooks $(22.5 \%)$, lectures $(21.8 \%)$, newspapers and media $(7.1 \%)$, other undesignated resources $(16.5 \%)$. The top known psychoactive and novel psychoactive substances were (Figure 5); cannabis, hashish, and synthetic cannabinoids (96.6\%), amphetamines and amphetamine-type stimulants (88.4\%), Ayahuasca (87.4\%), opium and opioid-related 
substances (85.9\%), Salvia Divinorum (82.9\%), valium (79.9\%), Xanax (79.2\%), shrooms and Psilocybin (77.7\%), Krokodil (56.7\%), Kratom (50.1\%), crystal Meth (49.5\%), Khat (34.3\%), NBOMe (33.8\%), captagon (14.1\%), Haldol (18\%), Olan (12.8\%), and octodrine (8.1\%).

The (ab)use of these substances was categorised based on the frequency of use into; few occasions per year $(33.4 \%)$, on a monthly basis (15\%), and on a weekly basis $(18.4 \%)$, while those who (ab)used NPS on a daily basis accounted for $24.2 \%$. In relation to chronicity of (ab)use, these were categorised into; few months (4.7\%), 1-5 year $(40.3 \%)$, more than 5 years $(14.6 \%)$, more than 10 years $(18.4 \%)$, more than two decades $(7.3 \%)$, and more than 30 years $(7.9 \%)$. The responders declared that they used to purchase substances from friends and family members $(66 \%)$ and from the internet via e-commerce $(7.9 \%)$. Some $(1.9 \%)$ were able to cock (design) their own substances of interest, while others (12.2\%) acquired their substances either from pharmacies via prescriptions, local chemists, or street dealers.

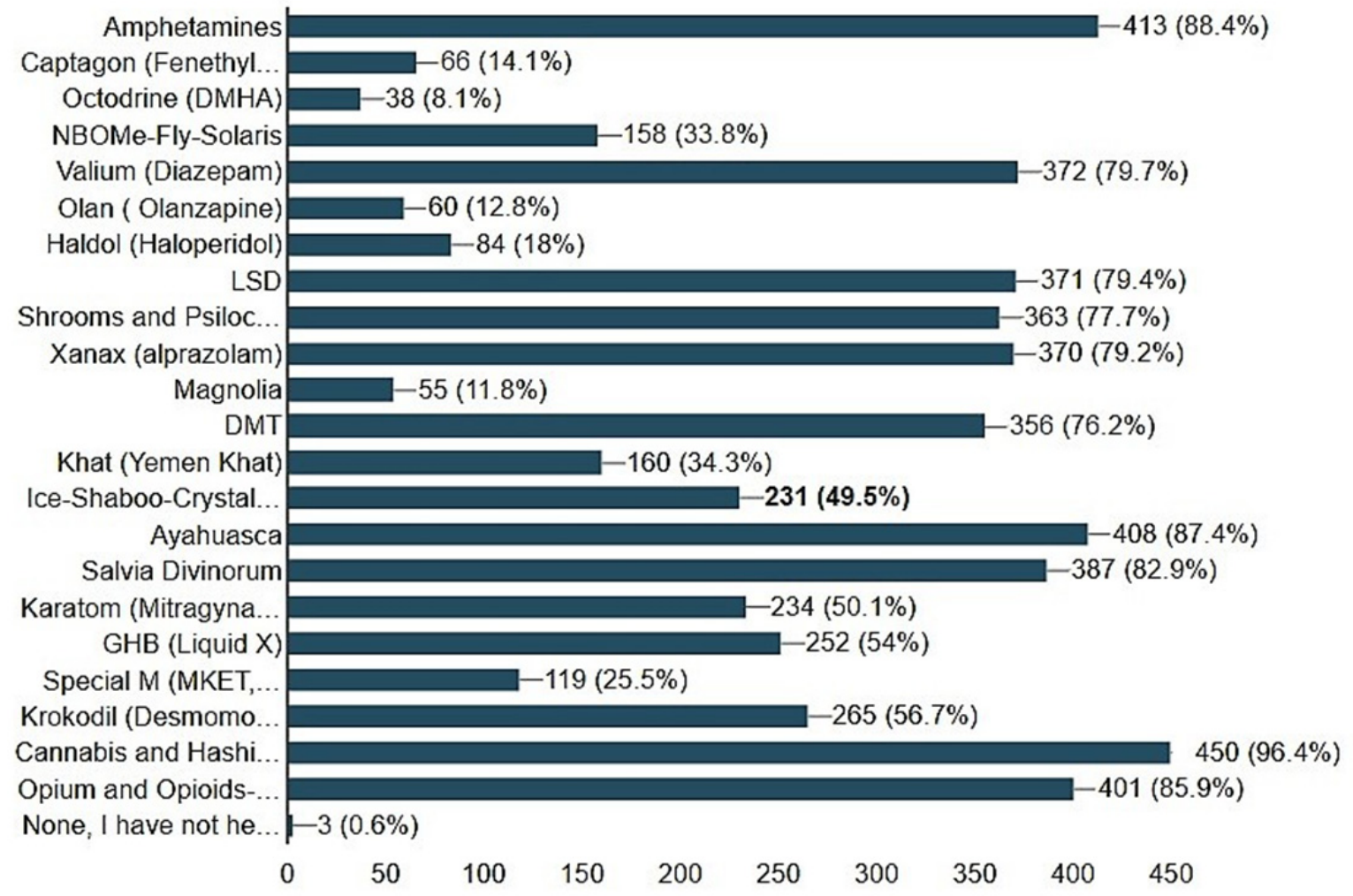

Figure 5. Mapped Knowledge of the Responders in Connection with Psychoactive Substances.

Additionally. Approximately, 67.9\% were aware of the presence of e-commerce activities. Only $7.3 \%$ of NPS (ab)users admitted to being taken to the hospital due to severe adverse reactions (Figure 6), these included; hallucinations $(66.2 \%)$, delusions $(33.6 \%)$, loss of consciousness $(18.2 \%)$, aggression and violent behaviour (7.3\%), hypersensitivity and allergic reactions (6.6\%), cardiac and vascular problems $(22.1 \%)$, respiratory problems including asthma and suffocation (4.7\%), musculoskeletal problems $(2.6 \%)$, epileptic seizures $(1.9 \%)$, and blood-transmitted diseases including AIDS (1.1\%). There was no statistically significant difference in age and gender among the top ten contributing countries of the Geo-map. On the other hand, there was a statistically significant difference in age between responders from the developed countries versus those from the Middle East ( $p$-value $=0.037)$; Middle Eastern responders were a bit older. It is possible that westerners are more of novelty seekers than those of the eastern culture in connection with psychoactive and novel psychoactive substances. However, the proportional contribution of males and females in between the two subpopulations (developed versus Middle Eastern) was not significantly different $(\mathrm{p}=0.44)$. 


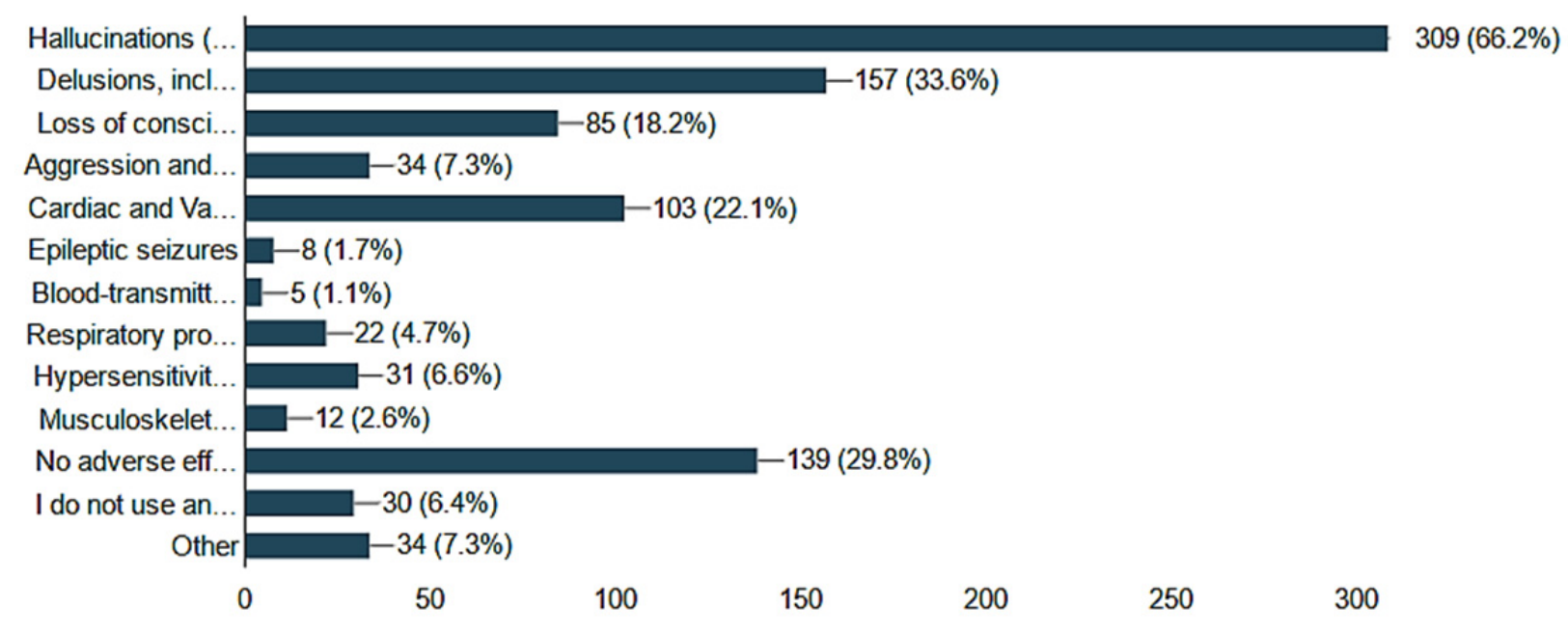

Figure 6. Most Commonly Encountered Adverse Reactions

\section{Discussion}

In the past two decades, designer drugs represented a significant evolving threat to health and economy; the phenomenon has been escalating at a logarithmic pace, paralleled only by the explosive collateral evolution within the discipline of information and communication technologies (Al-Imam et al., 2016; Al-Imam et al., 2017; Brew, 2016; Dargan \& Wood, 2013; Lin et al., 2014). The technologic advancement in the developed western world made it feasible for the NPS, its e-commerce, and the collateral e-phenomena on both divisions of the web, to flourish to unprecedented levels beyond the control of legal authorities and regulating bodies. Accordingly, the NPS phenomena are more diffused in the developing world (Al-Imam et al., 2016; Al-Imam et al., 2017; Bigdeli et al., 2013). In 2014, Best and colleagues studied the online platforms including the social media in connection with the adolescent well-being; their systematic review of the literature was based on assessing 43 original studies that were committed for the purpose of evaluating the harmful and beneficial effects of these online platforms. There was a contradictory evidence although it was concluded that online platforms and technologies could have a detrimental effect on health well-being and yet can also be exploited for health and social care purposes (Best et al., 2014). Few research attempts were dedicated to the NPS phenomenon in the developing world as in the case of the Middle East, Latin and Central America, Africa, and Asia (Dargan \& Wood, 2013).

This observational cross-section of a renowned social online platform represents one of the few attempts to analyse selected populations of virtually-placed individuals, and assess the proportional contribution of the developed versus the developing countries in connection with the extent of spread and (ab)use of psychoactive substances on the surface web. However, there have been prior attempts dating back to the end of the last decade (Al-Hemiary et al., 2010; Dargn \& Wood, 2013).

Al-Hemiary and colleagues have led one of the earliest attempts in Iraq. By 2012, they have concluded that the most commonly used substances were alcohol, cannabis, and prescription-related chemicals; Novel drugs (NPS) started to appear on the drug scene as in the case of amphetamine-type substances (ATS) including captagon and crystal (methamphetamine). Other traditional psychoactive substances were also found in abundance including; tramadol, Afghan opium, teriac (an opioid), and heroin (Al-Hemiary et al., 2010; Al-Hemiary et al., 2014; Al-Hemiary et al., 2016). Subsequent research attempts by Al-Imam and colleagues in 2016 has confirmed that captagon was infiltrating the destabilized countries of the Middle East; captagon was available in e-commerce within the UAE, Syria, Iraq, and Turkey; the substance was diffuse in a dozen of e-markets on the darknet, including AlphaBay, Agora, Valhalla, and Hansa (Al-Imam et al., 2017). Further, Al-Diwan and coworkers inferred that high household density (crowding density) and low educational status were correlated with alcohol and substance abuse; the peak of age for using psychoactive substances was found to be 24-35 years which is concordant with the data provided in our study (Al-Diwan et al., 2015). In 2013 and 2014, Bigdeli and colleagues discovered the existence of fourteen new substances; data were extrapolated from 104 websites. The majority of these substances were either herbal, synthetic, pharmaceutical, or combinatory chemicals that are promoted via the e-commerce (Bigdeli et al., 2014). 
Very recently (2017), Joh and co-workers have conducted cross-sectional analyses among Korean University students with an aim to assess the health promotion in young adults following a three-days university-wide health promotion program (Joh et al., 2017). The program was aiming at enhancing health awareness via promoting a healthy diet, healthy behaviour, and an active lifestyle and; the program was successful. An earlier attempt in 2012, Dargan and Wood have studied the recreational drug use, including those of traditional and novel psychoactive substances in the Asia Pacific region via critical analysis of the UNODC programmes of relevance to the topic; they have concluded that there are still gaps to be filled within these constructive programs (Dargan \& Wood, 2012).

\section{Conclusion}

This study is the $1^{\text {st }}$ of its kind to provide a statistical inference in relation to the level of knowledge, the extent of (ab)use, and the adverse reaction in connection with traditional and novel psychoactive substances. This study can serve as a blueprint for future studies comparing specific populations, for example, western versus Middle Eastern. Most of the survey participants were either from the US, UK, India, or western Europe. Perhaps, future studies may attempt to explore the differential effect of specific demographic parameters including the ethnic backgrounds and the religious affiliations in connection with psychoactive substances' use and misuse. Geo-mapping revealed a minimal contribution from the Middle East, the highest of which was observed in Tunisia and Turkey. Most of (ab)users were residents of urban areas, living either with parents or with a partner, and being unmarried; there was a relatively high prevalence of neuropsychiatric illnesses. There was also an advanced level of NPS awareness, (mis)use, involvement in e-commerce activities, and incidence of adverse reaction events including intoxication and hospitalization. Further, the frequency and chronicity of (ab)use were high. The NPS substance use and misuse represent a unique phenomenon that is more diffused in the advanced western world; the contribution of the developing countries, including the Arab world and the Middle East, appeared to be insignificant. However, this is to be further explored in future studies.

\section{Acknowledgments}

The authors would like to acknowledge the efforts of medical students at the College of Medicine-University of Baghdad; Abdallah Anmar, Narveen Geen, Zina Faisal, Abdullah Muhanned, Hussein Ali Albayati, Sara Osama Jabbar, Mohammad Saleh Ibrahim, Ammar Jalel Raad, Rawan Omar Farooq, Muhammed Tariq Mutar, and Zaid Emad Abed for their assistance in distributing the survey.

\section{Competing Interests Statement}

The authors have no conflict of interest to be declared.

\section{Source of Funding}

This study was entirely self-funded.

\section{References}

Al-Diwan, J., Al-Hemiary, N., \& Hashim, M. (2015). Alcohol and Drug Abuse in Post-conflict Iraq. European Psychiatry, 30, 1394. https://doi.org/10.1016/S0924-9338(15)31080-4

Al-Hemiary, N. J., Hashim, M., Al-Diwan, J., \& Abdulrazzak, E. (2010). Alcohol and drug abuse in post-conflict Iraq. Unpublished manuscript. Baghdad University, Iraq.

Al-Hemiary, N. J., Al-Diwan, J. K., Hasson, A. L., \& Rawson, R. A. (2014). Drug and alcohol use in Iraq: findings of the inaugural Iraqi Community Epidemiological Workgroup. Substance use \& misuse, 49(13), 1759-1763. https://doi.org/10.3109/10826084.2014.913633

Al-Hemiary, N.J., Hashim, F.M., Al-Shawi, C.A. (2016). Effect of post-traumatic stress disorder on school achievement among secondary school students in Baghdad, Iraq. J Fac Med Baghdad, 58(2).

Al-Imam, A. (2017). Could Hallucinogens Induce Permanent Pupillary Changes in (Ab) users? A Case Report from New Zealand. Case Reports in Neurological Medicine, 2017. https://doi.org/10.1155/2017/2503762

Al-Imam, A. (2017). The relation between cerebral dominance and visual analytic skills in Iraqi Medical students, a cross sectional analysis. Journal of the Anatomical Society of India, 66(1), 42-43. https://doi.org/10.1016/j.jasi.2017.08.136

AL-Imam, A., Santacroce, R., Roman - Urrestarazu, A., Chilcott, R., Bersani, G., Martinotti, G., \& Corazza, O. (2017). Captagon: use and trade in the Middle East. Human Psychopharmacology: Clinical and Experimental, $32(3)$. 
Al-Imam, A., Simonato, A. P., \& Corazza, O. (2016). Haloperidol, an old antipsychotic with potential use by NPS users in Iraq. Research and Advances in Psychiatry, 3(3), 81-84. Retrieved from https://www.rapjournal.eu/materiale_cic/948_3_3/8031_haloperidol/article.htm

Best, P., Manktelow, R., \& Taylor, B. (2014). Online communication, social media and adolescent wellbeing: A systematic narrative review. Children and Youth Services Review, 41, 27-36. https://doi.org/10.1016/j.childyouth.2014.03.001

Bigdeli, I., Corazza, O., Aslanpour, Z., \& Schifano, F. (2013). Novel psychoactive substances (NPS): A study on Persian language websites. Iranian journal of public health, 42(5), 511.

Brew, I. (2016). Novel psychoactive substances. The British Journal of General Practice, 66(644), 125. https://doi.org/10.3399/bjgp16X684253

Burns, L. (2014). World Drug Report 2013 By United Nations Office on Drugs and Crime New York: United Nations, 2013ISBN: $978-92-1-056168-6,151$ pp. Grey literature. Drug and Alcohol Review, 33(2), 216-216. https://doi.org/10.1111/dar.12110

Chou, W. Y. S., Hunt, Y. M., Beckjord, E. B., Moser, R. P., \& Hesse, B. W. (2009). Social media use in the United States: implications for health communication. Journal of medical Internet research, 11(4). https://doi.org/10.2196/jmir.1249

Dargan, P., \& Wood, D. (Eds.). (2013). Novel psychoactive substances: classification, pharmacology and toxicology. Academic Press.

Dargan, P. I., \& Wood, D. M. (2012). Recreational drug use in the Asia Pacific region: improvement in our understanding of the problem through the UNODC programmes. Journal of Medical Toxicology, 8(3), 295-299. https://doi.org/10.1007/s13181-012-0240-4

Facebook. Facebook. Retrieved 16 March, 2017, from https://www.facebook.com/

Facebook. Magic Mushroom (Psilocybin). Retrieved 16 March, 2017, from https://www.facebook.com/groups/1546595612323918/

Facebook. Psychedelic Experience. Retrieved 16 March, 2017, from https://www.facebook.com/groups/815158201837615/

Facebook. Psychedelic Heaven. Retrieved 16 March, 2017, from https://www.facebook.com/groups/Psychedelic.Heaven/

Facebook. Psychedelics \& Philosophy 2. Retrieved 16 March, 2017, from https://www.facebook.com/groups/1233956900000615/permalink/1315808575148780/?comment_id=1315 953378467633 $\neg$ if_t $=$ group_comment $\neg$ if_id $=148742705280730$

Facebook. The Adult Psychedelic Sweet Shop. Retrieved 16 March, 2017, from https://www.facebook.com/groups/763405830462403/

Facebook. The Terance McKenna Experience. Retrieved 16 March, 2017, from https://www.facebook.com/groups/terencemckenna/

Ferri, M., Bo, A., Amato, L., Guedes, I. C., Esteves, C. S., Wiessing, L., ... \& Davoli, M. (2015). What is needed in future drug treatment research? A systematic approach to identify gaps on effectiveness of drug treatment from the EMCDDA. Drugs: education, prevention and policy, 22(1), 86-92. https://doi.org/10.3109/09687637.2014.954988

Hirst, M., Harrison, J., \& Mazepa, P. (2014). Communication and new media: From broadcast to narrowcast. Oxford University Press.

Howick, J., Chalmers, I., Glasziou, P., Greenhalgh, T., Heneghan, C., Liberati, A., ... \& Thornton, H. (2011). Explanation of the 2011 Oxford Centre for Evidence-Based Medicine (OCEBM) levels of evidence (background document). Oxford Center for Evidence-Based Medicine. Retrieved from http://www. cebm. net/index.aspx .

Jensen, K. B. (Ed.). (2013). A handbook of media and communication research: Qualitative and quantitative methodologies. Routledge.

Joh, H. K., Kim, H. J., Kim, Y. O., Lee, J. Y., Cho, B., Lim, C. S., \& Jung, S. E. (2017). Health promotion in young adults at a university in Korea: A cross-sectional study of 625 participants in a university. Medicine, 96(7). https://doi.org/10.1097/MD.0000000000006157 
Lin, C. A., \& Atkin, D. J. (Eds.). (2014). Communication technology and social change: Theory and implications. Routledge.

Mackey, T. K., \& Liang, B. A. (2013). Improving global health governance to combat counterfeit medicines: a proposal for a UNODC-WHO-Interpol trilateral mechanism. BMC medicine, 11(1), 233. https://doi.org/10.1186/1741-7015-11-233

Moorhead, S. A., Hazlett, D. E., Harrison, L., Carroll, J. K., Irwin, A., \& Hoving, C. (2013). A new dimension of health care: systematic review of the uses, benefits, and limitations of social media for health communication. Journal of medical Internet research, 15(4). https://doi.org/10.2196/jmir.1933

Toohey, K., \& Beaton, A. (2017). International cross-sector social partnerships between sport and governments: The World Anti-Doping Agency. Sport Management Review. https://doi.org/10.1016/j.smr.2016.12.004

Wood, D. M., Hill, S. L., Thomas, S. H., \& Dargan, P. I. (2014). Using poisons information service data to assess the acute harms associated with novel psychoactive substances. Drug testing and analysis, 6(7-8), 850-860. https://doi.org/10.1002/dta.1671

\section{Copyrights}

Copyright for this article is retained by the author(s), with first publication rights granted to the journal.

This is an open-access article distributed under the terms and conditions of the Creative Commons Attribution license (http://creativecommons.org/licenses/by/4.0/). 